A recent study on mice has successfully transplanted stem cells into those with visual impairment. These

first skirmishes in the battle against blindness are cause for optimism for restoring sight in the blind.

\title{
Retinal stem cell research suggests hope is in sight
}

In a study funded by the M edical Research Council, scientists from the University College London Institutes of $O$ phthalmology and $\mathrm{C}$ hild $\mathrm{H}$ ealth and M oorfields Eye H ospital, UK, have successfully transplanted immature retinal stem cells into mice.

'...the scientists succ eeded by using more advance cells that were able to develop into photorec eptors.'

Previous studies have failed and it was believed that the mature retina had no capability to repair, but the scientists from the University of M ichigan, M I, USA, and London's Institute of $O$ phthalmology succeeded by using more advance cells that were able to develop into photoreceptors. The study involved transplanting cells from 3-5-dayold mice, a mimicked human retinal disease. The retinal cells were able to reorientate themselves after being injected and went on to form photoreceptors and electrical connections with theexisting retinal nerve cells. This is the key to restoring sight, and further tests demonstrated activity in the optical nerve and light sensitivity.

Stem cells from many organisms have the capacity to generate in vitrointo a

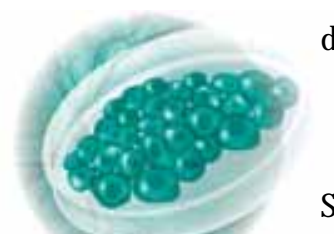

diabetes and heat failure. Recent advances made by the royal College of Surgeons indicate that retinal pigmented epithelial (RPE) cells exhibiting the characteristics of their natural counterparts can be induced from undifferentiated embryonic stem (ES) cells. Batches of RPE cells have been derived for use in an animal model of retinal disease.

The researchers cautioned it would be several years before similar efforts would be attempted in humans, although the report is described as "a stunning piece of research that in the distant future may lead to transplants in humans to relieve blindness." The study is the first of its kind to suggest people who have al ready lost crucial light-sensitive cells, through macular degeneration, can have their vision restored.

Restoring human blindness would involve taking stem cells from a fetus during the second trimester of pregnancy, so efforts are being taken to concentrate on using adult stem cells situated on the margin of the adult retina. These cells are identified as having stem cell like properties. Dr Robert M acLaren, a specialist at M oorfields Eye H ospital who worked on the research, said the aim now would be to look at adult stem cells to see if they could be genetically altered to behave like the mouse retinal cells.

“...a stunning piece of research that in the distant future may lead to transplants in humansto relieve blindness."

\section{blind ness."}

stage when

the retina is about

to be formed, into modified mice that
Stem cells promise to provide a wellcharacterized and reproducible source of replacement tissue for human clinical studies and are al ready showing large amounts of therapeutic success in the treatment of many other diseases, including 


\section{Wayward stem cells could be cause of brain tumors}

A novel approach to brain cancer is being explored by Steven Goldman, a Professor in the D epartment of N eurology and $C$ hief of $D$ ivision of $C$ ell and $G$ ene Therapy at Rochester M edical Center, NY, U SA.

"O verall the treatments for brain cancer aren't much better than they were decades ago," declared Goldman.T his has driven Goldman to approach different methods for treatment and, through this, he has receive an award from the foundation's 21st Century Science Initiative in Brain Cancer Research, which has provided funding. Current research is exploring the notion that the source of brain tumors could be rare 'bad' stem cells present in the brain. Stem cells are present in very small numbers in the brain and act as mast cells.
They are crucial to enable recovery from brain injury and are able to differentiate into almost any type of brain cell. The presence of 'bad' stem cells makes brain tumors particularly difficult to treat with chemotherapy, since the altered cells are present in large numbers compared with normal tumor cells.

Goldman was able to isolate specific cells in the 'biochemical brew' that is the brain and track their growth and differentiation into neural cells. G oldman is currently analyzing stem cells at different points of development, which enables him to distinguish factors effecting stem cell differentiation, and he hopes to be able to pinpoint what is crucial in determining a stem cell's fate. By comparing the factors that make heal thy stem cells with those involved in the formation of their 'bad' tumorcausing counterparts, identification of the determining factor or factors may be possible. If the identification of these genes is successful, it could start a whole new type of gene therapy for brain tumors. Already, the identification of one molecule hasled to hope in treating a rare type of brain cancer known as neurocytoma.

This research could have effects in all areas of cancer, and Goldman is positive that it will greatly improve our understanding of other disease, such as H untington's disease, Parkinson's disease, multiple sclerosis and several rare but fatal forms of neurodegenerative disease in children.

\section{H eart attacks - stem cells defend}

A new stem cell therapy designed to beused within hours of a cardiac arrest is due to undergo clinical trials at two leading London hospitals, UK. The revolutionary treatment involves the use of a patients' own stems cells extracted from the bone marrow in the hip and is the first to be supported by the UK Stem C ell Foundation. Previous research using animal and clinical studies indicate that the treatment will work and could enable recovery and repair of the heart muscle after a heart attack.

ES cells have been used in research since 1981, when they were isolated from mice, due to speculation of their therapeutic properties. 1998 saw the isolation of the first human stem cell in the USA and this sparked the need for the introduction of the H uman Reproductive Cloning Act 3 years later. In 2005, scientistsin California, U SA, found that injecting human neural stem cellsappeared to repair spinal cordsin mice. This breakthro ugh paved the way for further investigation into regeneration of other tissues, and preceded the start of the UK stem cell bank in 2004.

$\mathrm{H}$ eart attacks or Acute myocardial infarction (AMI) account for 460,000 deaths in the USA each year. AMI is usually caused by coronary thrombosisand can lead to full cardiac arrest, causing damage to the heart tissue and increasing the chance of a second heart attack; survivors often go on to diefrom longterm effects, including heart failure. The $\mathrm{N}$ ational Women's $\mathrm{H}$ ealth Information Center claims that $24 \%$ of men die within a year of a heart attack in the USA.

Clinical trials in Germany demonstrated that the technique was safe, although the stem cells were administered late on during the heart attack, which is thought to greatly reduce the chances of success. N ow scientists aim to provide evidence of tissue repair when cells are administered within $5 \mathrm{~h}$ of the onset of a heart attack. $\mathrm{N}$ ot only is it speculated that tissue repair will occur, but John $M$ artin, a cardiologist with the British $\mathrm{H}$ eart Foundation, said the treatment, given in conjunction with routine angioplasty in cases of acute heart attacks, could even prevent the tissue damage.

In total, 100 patients will beinvolved in the study, with 50 receiving angioplasty to remove blockage to the artery, followed by an injection into the artery of the patients' own stem cells, within $5 \mathrm{~h}$ of diagnosis.
The remaining participants will receive angioplasty with a placebo injection. Follow-up examinations will take place after 2 years, using M RI scans to assess the condition of the heart, and quality-of-life assessments will be used to determine the success of the trial.

"C ardiac stem cell therapy is on top of our agenda," says LifeC ell vice chairman $\mathrm{S}$ Abhaya Kumar. Several factors make this therapy an attractive method of treating heart disease. The stem cells avoid the ethical debate surrounding much of its related research, since they are harvested from the patients own bone marrow. This also means that the likelihood of rejection and, hence, further complications is much lower. M oney is al so a reassuring factor, as this potentially life-saving treatment carries virtually no extra cost. T he treatment will not involve a longer hospital stay for the patient and no expense is required in the use of novel drugs and their development.

This research is being wel comed by Professor Weissberg, M edical Director of the B ritish $\mathrm{H}$ eart Foundation. W iessberg commented that "stem cells could herald a new frontier for heart patients." 


\section{Stem cells participate in two-way cellular communication}

Scientists from The University of Washington, WA, USA, have demonstrated that stem cells use two-way cellular communication. This dialog occurs in small areas in the body that coincide with stem cell differentiation. These so-called 'niches' are constructed of regulatory cells, allowing the cells to form an environment in which they can self renew and supply the body with the correct amount and type of required cells.

In the regulatory areas, for example, if cell production accelerates it could cause malignancies, and if too few cells are produced, degeneration of the body will occur and maintenance will fail; this could be the key to how our bodies age. Ruohola-Baker from the research team explained, "D emonstrating that stem cells can contribute to niche function has farreaching consequences for stem cell therapies and may provide insight on how cancer might spread throughout the body via populations of cancer stem cells." She also added that "stem cells hold high hope in regenerative medicine."

Stem cells have the ability to self renew by division to allow repair to damaged tissues, and this process must be regulated to keep the body maintained and to stop accelerated production, which could lead to cancer. The research has focused on signaling between regulatory cells and stems cells in these small areas. The notch signaling pathway was investigated. This is a pathway that involves a protein trigger attached to the cell that, when activated by delta and serrate proteins, recruits secondary messen gers able to mask or activate genes in the cell nucleus. U sually, this pathway depends on direct contact between cells, and the research indicated that the pathway plays an important role in many stem cell niches.
Increases in delta protein production in the stem cells or the presence of activate notch protein in the surrounding niche cells resulted in atenfold increase of niche cells, which consequently caused an increase in stem cells.

"O ur study now shows that stem cells use the $\mathrm{N}$ otch pathway to signal to neighboring cells to maintain an active niche, and in turn, the niche induces and maintains the fate of the stem cells," said Ruohola-Baker. The indication of the two-way dialog comes from the finding that the trigger for the notch pathway is present in the niche cells, meaning they not only send messages to stem cells but also receive them. It is speculated that this research may indicate the existence of multiple niches for specific stem cell differentiation that can be activated when stem cells are present, via the two-way dialog.

\section{Mechanical forces push stem cells to differentiate}

Supported by the $\mathrm{N}$ ational Institute of $\mathrm{H}$ ealth, bioengineers from the University of California, CA, USA, have managed to push bone marrow stem cells towards becoming blood vessels.

Embryonic stem cells are easy to work with and have the advantage of being able to differentiate into any kind of body cell; however, ethical problems plague further research and advancements. Research has shown that mesenchymal stem cells also have the ability to turn into different types of connective tissue, including bone, cartilage and muscle. Both types of cells hold promise for disease treatment.

The bioengineers investigated the role of the physical environment on mesenchymal cell development by observing the effect of mechanical forces.
M esenchymal cellswere grown on silicone membranes with grooves and were stretched longitudinally every second, on a device known as a 'stretch chamber.'

\footnotetext{
"Stem cells seem to know the type of tissue they a re supposed to become by the type of mechanic al stra in they a re subjected to."
}

Song Li, U C Berkeley Associate Professor of bioengineering and principal investigator of the study explained that "the mechanical effects on the body are well known. A good example is when astronauts in space experience a loss of bonemass becausethereis no gravity." The stretching of the cells every second mimics an average adult heart beat. After 2 days, the researchers found a significant increase in the expression genes that control tensile strength. These genes are important in producing tissuethat can withstand forces, such as those in vascular walls. Genes associated with cartilage and bone tissue, which are required to be compression bearing, were seen to decrease in the cells. The findings appeared to indicate that the stem cells were on their way to becoming smooth muscle tissue cells, although this was not quite achieved.

"Stem cells seem to know the type of tissue they are supposed to become by the type of mechanical strain they are subjected to," concluded Li. To achieve complete cell differentiation in a laboratory it is likely that a combination of chemical and mechanical factors will be required. 


\section{$\mathrm{N}$ ew gene aids understanding of kidney development}

The discovery of a key gene named Six2 has aided scientists' understanding of how our kidneys develop.

Researchers from St Jude children's Research H ospital found that the gene plays a critical role in regulating the population of stem cells available for specific parts of kidney development. These mesenchymal blastemal stem cells are available to differentiate into cells that form nephrons. Thenephronslater become attached to duct tubes that collect the filtered blood as urine, before the urine is excreted.

Guillermo O liver, who was involved in the research, said " $O$ ur discovery of the role of Six2 in the developing kidney suggests that a similar mechanism exists in other developing organs."

The research involved using knockout mice lacking the Six 2 gene, and results showed that they produced abnormal numbers of nephrons that did not go on to develop correctly, causing a mass of useless tissue that collect in the kidney. The stem cells present underwent apoptosis, which drastically reduced the number of cells available for kidney development. The researchers al so found that SiX2 gene suppresses a cascade of interactions normally triggered by a gene called Wnt4, which usually drives the normal development of kidneys.

'Researchersfound that the gene plays a critic al role in regulating the population of stem cells available for specific parts of kidney development.'

"O ur work shows that Six2 is critical to preventing the developing kidney from running out of stem cells and collapsing into a mass of underdeveloped tissue," Guillermo added.

\section{Mutations in PLCE1 gene found to play a role in early-onset nephritic syndrome}

Using positional cloning, scientists from the University of M ichigan (UM ) have identified recessive mutations in the phospholipase $\mathrm{C}-\varepsilon$ gene (PLCE1), which is known to cause early-onset nephrotic syndrome with end-stage kidney disease.

Early-onset nephritic syndrome causes kidney filtering units called glomeruli to fail to develop normally. This leads to impaired waste excretion from the blood and loss of important proteins. Proteins leaking out from the glomerulus are excreted in urine and body tissues retain too much water, causing swelling around the eyes. "T he early onset form of the disease is severe and infants often suffer from end-stage renal disease within the first year of life," described Friedhelm H ildebrandt, Director for the $C$ ure and Prevention of Birth D efects at the U M . In children with the disease, developing glomeruli appear smaller and lack structural complexity. The key proteins, such as nephrin, responsible for glomerulus function, are not expressed.
Using immunflourecence, the scientists found that the PLCE1 gene was expressed during the development and maturation of glomerular podocytes. Without normal glomerular development, diffuse mesangial sclerosis (DMS) occurs, which is the presence of lesions on the glomerlurus. These lesions cause the kidney to progress rapidly to end-stage kidney failure.

Some types of nephrotic syndrome can be treated with steroids or other drugs, although steroid-resistant forms of the disease as a rule do not respond to treatment, and the disease progression is very quick in infants, with most exhibiting end-stage renal failure before the age of 5 years. "Until now, most physicians believed there was no point in trying treatment," commented $H$ ildebrandt.

PLCE1 is not the only gene involved in steroid resistant, in fact, it is the seventh to be discovered and the second to be associated with specialized cells, podocytes, which surround the capillaries and influence glomerular development. The majority of PLCE1 mutations prevent normal podocyte development in the embryo.

Blood samples from 12 children with inherited recessive mutations of PLCE1, resulting in a diagnosis of steroidresistant forms of the disease, were analyzed. The PLCE1 gene mutation was identified, and all children received treatment with steroids or cyclosporine A. A total of five children progressed to end-stage renal failure but, surprisingly, two children survived, becoming healthy and now show no symptoms of the disease. Hilderbrand believes that there may be a "critical time window during which treatment can overcome the development defects caused by PLCE I's loss of function."

Future research is planned to continue searching for the causes of nephrotic syndrome and to use the Z ebrafish model with high-throughput drug screening, to identify drugs capable of reversing the kidney damage. 


\section{in brief. .}

Surface functionalization of inorganic nano-crystals with fibronectin and E-cadherin chimera synergistically accelerates trans-gene delivery into embryonic stem cells.

Kutsuzawa K, Chowdhury EH, Nagaoka M, Maruyama K, Akiyama Y, Akaike T: Biochem. Biophys. Res. Commun. 350(3), 514-520 (2006).

This study describes the development of a biofunctionalized inorganic nanocarrier of DNA, which can be used for gene transfer to stem cells. Fibronectin and E-cadherin chimera were embedded on the carrier, resulting in high-affinity interactions with the embryonic stem cell surface and accelerated transgene delivery for subsequent expression.

Fibronectin-anchored particles and, to a greater extent, fibronectin and E-cadherin- $\mathrm{Fc}$ associated particles enhanced transgene delivery dramatically, with a value higher than that of commercially available lipofection systems. Conventional methods of transferring genes to stem cells suffer from various disadvantages. These findings could lead to the establishment of a biofunctional hybrid gene-carrier for use in developing stem cellbased therapy in regenerative medicine.

Grow th of mesenchymal stem cells on electrospun type I collagen nanofibers. Shih YR, Chen CN, Tsai SW, Wang YJ, Lee OK: Stem Cells 24(11), 2391-2397 (2006).

The morphology, growth, adhesion, cell motility and osteogenic differentiation of human bone marrow-derived mesenchymal stem cells (M SCs) were examined when cultured on Type I collagen nanofibers of three nanosized diameters (50-100, 200-500 and 500-1000 nm). Cells on the nanofibers showed more polygonal and flattened morphology and $\mathrm{MSC}$ s grown on 500-1000-nm nanofibers had significantly higher cell viability than the tissue culture polystyrene control. There was no difference after 12 days of osteogenic differentiation and there was also comparable osteogenic gene expression of osteocalcin, osteonectin and ostepontin between cells differentiated on polystyrene and nanofiber surfaces. The results suggest that Type I collagen nanofibers support the growth of MSCs without compromising their osteogenic differentiation capacity. These nanofibers could also be used as a scaffold for bone tissue engineering to facilitate intramembranous bone formation.

\section{M ystery of Z ebrafish heart regeneration solved}

Zebrafish posses the unique ability to regenerate cardiac tissue and, until recently, the mechanism of regeneration was poorly understood. Scientists from D uke University Medical C entre in D urham, NC, USA, discovered that when parts of a Zebrafish's heart were removed, stem cells start to play an important role in its regeneration.

"If you look in nature, there are many examples of different types of organisms, such as axolotls, newts and zebrafish, that have an elevated ability to regenerate lost or damaged tissue," said Kenneth Poss. The research led by M ark T Keating hopes to providea model that may givean insight into the regenerative potential in mammals. Some species have the ability to regenerate appendages when closely related species do not and, because of this, Poss speculates that our ability to regenerate may have been lost through evolution, and claims that "T he key is to find a way to turn on' this regenerative ability." This could lead to the discovery of novel treatments for heart disease and damage, and even limb regeneration.

The research indicated that stem cells form in the wound and in layers surrounding it, which encourages the regeneration. Also important is the discovery of specific growth factors that interact with the stem cells and a protective cell layer that covers the wound. First, a blastma is formed, this is a mass of undifferentiated stem or progenitor cells that have the potential to differentiate. W ithin the first 3-4 days after injury, these cells differentiate and proliferate. $\mathrm{N}$ ext, cardiac tissue surrounding the heart chambers induces developmental markers and expands, creating a new cell layer that covers the exposed heart tissue. Some of the cells then undergo epithelial to messenchymal transition (EM T.) These cells invade the wound and provide a new blood supply to regenerating muscles. This is described as making a "protective niche where the new heart muscle can grow."

Involvement of fibroblast growth factors (FG Fs) is suspected, when experimentally blocked by expression of a dominant-negative FG F receptor, EM T and coronary formation of a new blood supply fail. By understanding stem cell involvement and growth someday benefit millions of peoplewho suffer heart attacks or cardiac injury, allowing tissue regeneration instead of the formation of scar tissue, which impairs the heart's ability to pump and can lead to life-threatening arrhythmias.

"Scientists previously had suspected that zebrafish regenerated their heart tissue by the direct division of existing cardiac muscle cells adjacent to the injury," said Poss, but now this is known to not be the case. factor recruitment, this research might

\section{About the Bulletin Board}

The Bulletin Board highlights some of the most important events and launches in stem cell research and regenerative medicine. The editorial team welcomes suggestions for timely, relevant items. If you have newsworthy information, please contact:

Elisa Manzotti,

Editor, Regenerative Medicine,

Future Medicine Ltd, Unitec House,

2 Albert Place, Finchley Central, London N3 1QB, UK

E-mail: e.manzotti@futuremedicine.com

Tel: +44 (0)20 8349 2033; Fax: +44 (0)20 83432313 